\title{
A New Mathematical Model of High Speed Satellite Channel
}

\author{
Zheng Xiaotian, Li Jilin, Lin Mo, Wang Jian, and Li Xinhua
}

\begin{abstract}
A new mathematical model of satellite channel was proposed. On the basis of the analysis of high-speed remote sensing satellite link, and combining vector network test results, we obtained a new mathematical model of a high-speed satellite link. Through simulation analysis, the amplitude-frequency characteristics and group delay characteristics of the model is corresponded with the actual link. The model laid the foundation for analysis of future high-speed data transfer between satellite and ground.
\end{abstract}

Index Terms-High speed, satellite link, amplitude frequency characteristics, group delay.

\section{INTRODUCTION}

Due to the rapid growth of the mass of information, put a higher demand for satellite and ground data transfer rate. When the satellite data rate exceeded $100 \mathrm{Mbps}$, the Intersymbol Interference (ISI) and group delay will have a serious impact on SNR of the satellite link. In this paper, based on the analysis of a remote sensing satellite link, we build the set of platform of satellite link, its amplitude -frequency and group delay performance has been tested by experiment. We obtain a mathematical model of the satellite link with combination of theoretical analysis and experiment. The simulation results show that the model and the actual satellite links are in good agreement, which laid the foundation for the further study in high-speed data transmission.

\section{The Analysis of High SPeEd SATEllite ChanNel}

As shown in Fig. 1 is the remote sensing satellite link to the general, mainly including all the front-end equipment from modulator and demodulator and free space link. The link includes inverter, filters, amplifiers, cable and frequency converter, the signal is the main reason for the distortion.

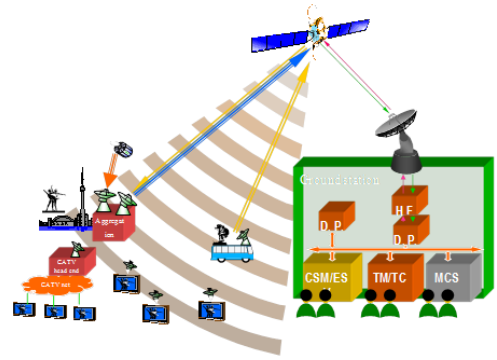

Fig. 1. Satellite link diagram.

Manuscript received January 10, 2013; revise April 22, 2013.

The authors are with Space Star Technology Co., Ltd. (SSTC), BeiJing, China (e-mail: zhengxt_paper@163.com).

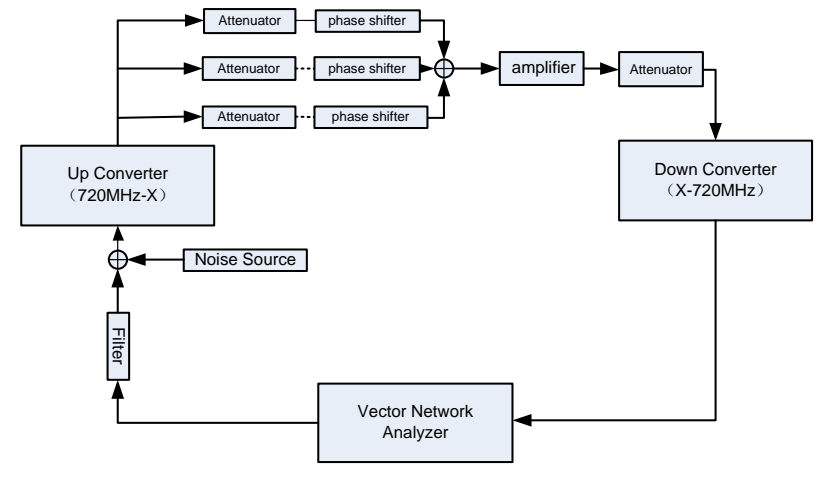

Fig. 2. Satellite link structure.
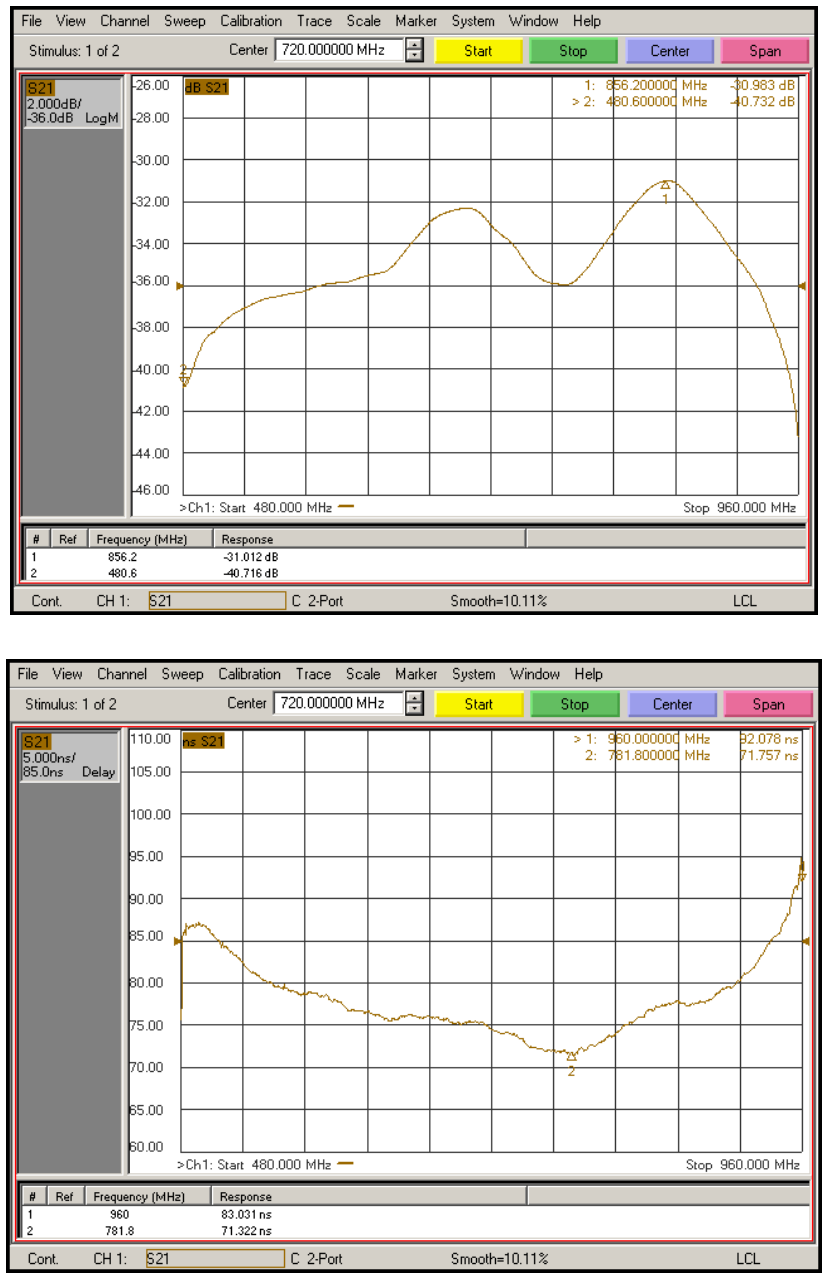

Fig. 3. Amplitude frequency (above) and the group delay (below) test results

From the above diagram we can obtain as shown in Fig. 2 of the satellite link structure. In order to simulating the link BER we add noise in the link[1]. Through adding noise to link Using three adjustable attenuators, different length of 
cable and adjustable phase shifter, we can simulate the amplitude distortion and the group delay characteristics of different level. This paper uses AgilentE8362B vector network analyzer test the link of the amplitude frequency characteristics and group delay characteristics [1]. The results as shown in Fig. 3, centered on $720 \mathrm{MHZ}$ frequency, bandwidth of $480 \mathrm{MHZ}$. As shown in Fig. 4, the BER of 480Mbps QPSK signal lost 7dB energy after the link.

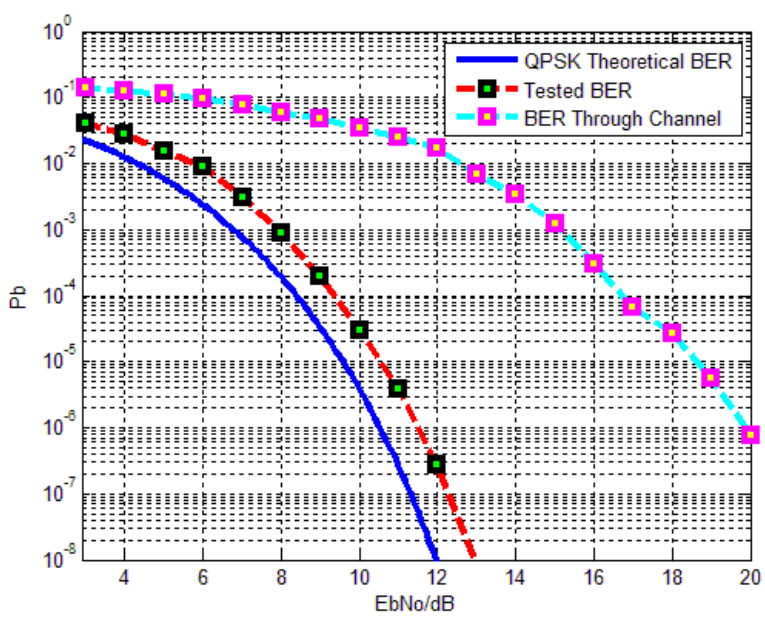

Fig. 4. BER through satellite channel.

\section{A New Mathmatical Model of Satellite Channel}

\section{A. Link Modeling and Parameter Solving}

In general, any channel response obeys the following equation:

$$
C=A(f) e^{-j 2 \pi \phi(f)}
$$

where, $A(f)$ represents channel amplitude-frequency characteristics, it is a function of frequency; $\phi(f)$ represents the phase-frequency characteristics, it is also a function of frequency[2]. The satellite link model is based on this function. Definition:

$$
\begin{aligned}
& A(f)=A\left[a_{0}+a_{1}\left(f-f_{0}\right)+a_{2}\left(f-f_{0}\right)^{2}\right. \\
& +a_{3}\left(f-f_{0}\right)^{3}+a_{4}\left(f-f_{0}\right)^{4}+a_{5}\left(f-f_{0}\right)^{5} \\
& \left.+a_{6}\left(f-f_{0}\right)^{6}\right]
\end{aligned}
$$

where, $f_{0}=768 \mathrm{MHz}$ represents a fading center frequency. Fig. 3 vector network test data substituted into (2) the above coefficients can be obtained by solving the matrix equation:

$$
\begin{aligned}
& A=0.857 \\
& a_{0}=-39.3460628071175 \\
& a_{1}=-0.111623494642515 \\
& a_{2}=0.00131288377366987 \\
& a_{3}=2.30251364930612 \mathrm{e}-05 \\
& a_{4}=-2.71872271914403 \mathrm{e}-08 \\
& a_{5}=-1.03725102648957 \mathrm{e}-09 \\
& a_{6}=-2.70834370456597 \mathrm{e}-12
\end{aligned}
$$

Obtained:

$$
\begin{aligned}
& A(f)=0.857 \times[-39.3460628071175 \\
& -0.111623494642515 \\
& \times\left(f-f_{0}\right)+0.00131288377366987 \times\left(f-f_{0}\right)^{2} \\
& +2.30251364930612 \mathrm{e}-05 \times\left(f-f_{0}\right)^{3} \\
& -2.71872271914403 \mathrm{e}-08 \times\left(f-f_{0}\right)^{4} \\
& -1.03725102648957 \mathrm{e}-09 \times\left(f-f_{0}\right)^{5} \\
& \left.-2.70834370456597 \mathrm{e}-12 \times\left(f-f_{0}\right)^{6}\right]
\end{aligned}
$$

Because of the relationship between group delay and phase signal are as follows[3]:

$$
\tau(f)=-\frac{d \phi(f)}{d f}
$$

The following anti-launch phase frequency expression by the group delay characteristics. Defined group delay[4]:

$$
\begin{aligned}
& \tau(f)=-b_{1}-2 b_{2}\left(f-f_{c}\right)-3 b_{3}\left(f-f_{c}\right)^{2} \\
& -4 b_{4}\left(f-f_{c}\right)^{3}-5 b_{5}\left(f-f_{c}\right)^{4}
\end{aligned}
$$

where the second is linear distortion, it is a linear function of a third and $\left(\omega-\omega_{c}\right)$ is proportional to, so called distortion of the square (parabola), the order components above the fourth small contribution to distortion, generally can be ignored[2]. Group delay test results into (5), can be obtained by solving the matrix equation:

$$
\begin{aligned}
& b_{1}=-75 \\
& b_{2}=0.0159722222222222 \\
& b_{3}=-2.65239197530866 \mathrm{e}-005 \\
& b_{4}=-3.01408179012347 \mathrm{e}-007 \\
& b_{5}=-6.27933706275685 \mathrm{e}-010
\end{aligned}
$$

Group delay model can be obtained as follows:

$$
\begin{aligned}
& \tau(f)=75-0.0319444444444444 \times\left(f-f_{c}\right) \\
& +7.95717592592598 \mathrm{e}-005 \times\left(f-f_{c}\right)^{2} \\
& +1.20563271604939 \mathrm{e}-006 \times\left(f-f_{c}\right)^{3} \\
& +3.13966853137842 \mathrm{e}-009 \times\left(f-f_{c}\right)^{5}
\end{aligned}
$$

By the (4) and (7) can obtain the phase model.

$$
\begin{aligned}
& \phi(f)=-75 \times\left(f-f_{c}\right)+0.0159722222222222 \\
& \times\left(f-f_{c}\right)^{2}-2.65239197530866 \mathrm{e}-05 \\
& \times\left(f-f_{c}\right)^{3}-3.01408179012347 \mathrm{e}-07 \\
& \times\left(f-f_{c}\right)^{4}-6.27933706275685 \mathrm{e} \\
& -10 \times\left(f-f_{c}\right)^{5}
\end{aligned}
$$

By the (3) and (8), the mathematical expression of the link can be obtained[5]: 


$$
\begin{aligned}
& C=A(f) e^{-j 2 \pi \phi(f)} \\
& =0.857 \times[-39.34606280-0.1116234946 \\
& \times\left(f-f_{0}\right)+0.00131288377 \times\left(f-f_{0}\right)^{2} \\
& +2.30251364930612 \times\left(f-f_{0}\right)^{3} \\
& -2.7187227191 \mathrm{e}-08 \times\left(f-f_{0}\right)^{4} \\
& -1.0372510264 \mathrm{e}-09 \times\left(f-f_{0}\right)^{5} \\
& \left.-2.70834370456 \mathrm{e}-12 \times\left(f-f_{0}\right)^{6}\right] \times \exp \{ \\
& -j 2 \pi\left[-75 \times\left(f-f_{c}\right)+0.015972222 \times\left(f-f_{c}\right)^{2}\right. \\
& -2.65239197 \mathrm{e}-05 \times\left(f-f_{c}\right)^{3}-3.0140817 \mathrm{e}-07 \times\left(f-f_{c}\right)^{4} \\
& \left.\left.-6.279337062 \mathrm{e}-10 \times\left(f-f_{c}\right)^{5}\right]\right\}
\end{aligned}
$$

\section{B. Model Simulation Results and Error Analysis}

By (3) available models of amplitude frequency, characteristic of the simulation result is shown in Fig. 5:

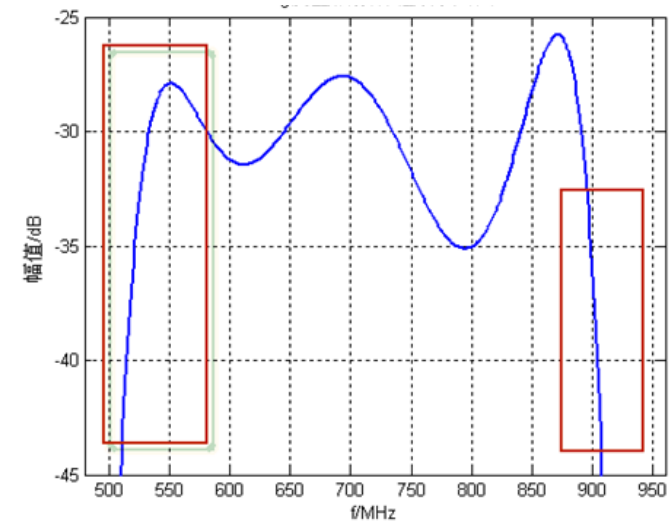

Fig. 5. The simulation results of link model of amplitude frequency characteristics.

Analysis of Fig. 3 and Fig. 5 may be found that the amplitude-frequency model fading center frequency, the maximum fade depth, and amplitude flatness is very close to the actual channel. Just near the band edge at the ups and downs more violent, which is the very nature of the decision by polynomial fitting[6].

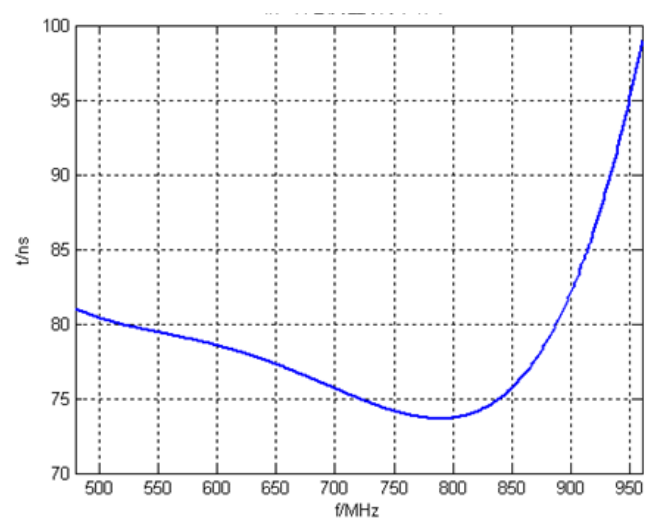

Fig. 6. The simulation results of the model group delay.

The equation (7) represents the group delay model simulation results shown in Fig. 6. Comparative Analysis of Fig. 3 and 6 can be found by the group delay model results obtained with the actual test results obtained in the approximate shape is the same, i.e. the group delay model is basically able to reflect the group delay of the real case[7]. The model is the use of polynomial fitting method to approximate the actual group delay, so there must be some differences in some details, but this difference is determined by fitting model. The observation model coefficient, its value is very small. Even further increase the order of the polynomial, and can not increase the accuracy of the model.

\section{CONCLUSION}

In this paper, obtaining a new satellite link model through the theoretical analysis based on solving the satellite link model parameters and experimental tests. Through simulation analysis, the amplitude-frequency characteristics and group delay characteristics of the model is very close link with the actual satellite link test results, to better reflect the satellite link characteristics. The model laid the foundation for the future of satellite high-speed data transmission analysis.

\section{REFERENCES}

[1] A. Coskun and I. Kale, "Blind correlation-based DFE receiver for the equalization of single input multi output communication channels," IEEE, 2009.

[2] D. C. Park, Y. S. Song, and D. M. Woo, "Equalization of 8PSK signals with a recurrent neural network," Advances in Neural Networks, pp. 105-110, 2007.

[3] A. A. Rontogiannis and K. Berberidis, "Bandwidth efficient transmission through sparse channels using aparametric channel-estimation-based DFE," IEEE Proc-Commun, vol. 152, no. 2, pp. 251-257, 2005.

[4] C. S. Khalaf and J. W. Stoughton, Design of infrasound-detection system via adaptive LMSTDE algorithm, 1984.

[5] C. E. A. Shannon, "Mathematical theory of communication," The Bell System Technical Journal, vol. 27, pp. 379-423 and 623-56, 1948.

[6] Y. Yi, R. Woods, L. K. Ting, and C. F. N. Cowan, "High speed FPGA-based implementations of delayed-LMS filters," Journal of VLSI Signal Processing 2005, vol. 39, pp. 113-31.

[7] R. Zeidler, A. Batra, J. R. Zeidler, and A. A. Beex, "Initialization techniques for improved convergence of LMS DFEs in strong interference environments," IEEE, 2007, pp. 3068-72.

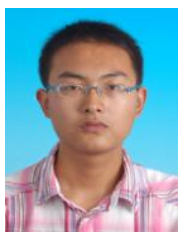

Zheng Xiaotian was born in China in 1986. He received his MSc in satellite communications and information system from China Academy of Space Technology (CAST) in 2008. Now he is a PhD candidate from CAST and Space Star Technology Co., Ltd. (SSTC).

Li Jilin was born in Hebei Province, in 1963. He received a M.Sc (Eng) in electrical engineering from Harbin Institute of Technology (HIT), Harbin, Beijing in 1983. From September 1983 until now, he worked at Beijing Institute of Satellite Information Engineering as engineer, senior engineer, Senior Research Fellow (Professor). As the Vice President of Space Star Technology Co., Ltd from 2004, Prof. LI is interested in design of satellite application system, integrated remote sensing processing system and satellite communication technology.

Lin Mo was born in Harbin, in 1981. He received the B.Sc (Eng.) in electrical engineering from Harbin Institute of Technology (HIT), Harbin, China in 2003. And He received the M.Sc (Eng) and Ph.D degree in Spacecraft Engineering from China Academy of Space Technology, Beijing, in 2009 respectively. He joined China Academy of Space Technology, Space Star Technology Corporation., Ltd in 2009 as the system engineer of SAR/Galileo EEV and MEOLUT project which are significant Sino-European cooperation in Galileo Satellite Navigation System. His research interests include SAR signal processing for development of novel signal processing algorithm and design of satellite application system. 
Wang Jian is a senior engineer from China Academy of Space Technology (CAST). He received his $\mathrm{PhD}$ of Information and Signal Processing in Beijing Institute of Technology in 2007. He is currently working in Space System Engineering, direction on satellite communication and navigation application technologies.

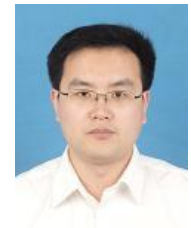

Li Xinhua received the B.S. degree in Electronics Engineering from Dalian University of Technology, China in 2001, and the M.S. degree in Aircraft design engineering from China Academy of Space Technology, China in 2004, where he was involved with system level design and simulation for the future generation of wireless communication systems. After that, he joined the Space Star Technology Co., Ltd .His research interests include satellite communication, spread spectrum communications, multiuser detection and advanced digital signal processing. 